\section{The Screening Review System: Fair or Foul?}

In March 1992, The Journal of Clinical Investigation (JCI) moved its editorial offices to La Jolla. This was the outcome of a nationwide search in which detailed applications were required of prospective editorial boards. One major issue candidates were asked to confront was the review process. The $J C I$ had a long tradition of rigorously and completely reviewing all submissions. However, this was not well suited to the pace of modern scientific publishing and to the expectation of authors for more rapid decision making. Furthermore, it was necessary to deal with the increased volume of submissions, which had continued unabated since 1989 , when several changes were introduced by the Bay Area editors (1). The challenge was to develop a new approach which would accelerate the speed of the review process without compromising its quality, while also lessening the burden on reviewers. The La Jolla group suggested several changes in the running of The Journal (2), many of which were subsequently implemented $(3,4)$. Of all these, perhaps the most unusual (and consequently, the most controversial) has been the implementation of a novel screening review process for manuscripts (2-4).

The screening review system: how and why? Past editors and experienced reviewers have indicated that many manuscripts are recognizable at the outset as having little hope of achieving priority for acceptance, regardless of their scientific quality. In principle, it would therefore be best for authors, reviewers, and editors if such manuscripts could be rapidly and accurately identified, allowing an early decision. This, however, would have to be done without compromising the fairness and objectivity that is the cornerstone of the peer review process $(5,6)$. Some other prestigious journals were already using a variety of rapid screening techniques, including informal decision making by the editors or formal screening by a special panel of reviewing editors (7). The $J C I$ board felt that editors of a broad-based non-specialty journal, however learned and wise they might be, could not accurately judge the lack of "importance" and "broad interest" of a submitted article, unless it happened to fall directly within their own immediate fields of expertise. Thus, attempts by editors to quickly identify the "better" articles at the outset could backfire, making The Journal a home only to fields in which the editors happened to have an interest.

The $J C I$ editors have therefore chosen to reject without external review only the small fraction of articles $(\sim 10 \%)$ that are either $(a)$ obviously deficient in scientific approach or content or $(b)$ happen to fall directly within the expertise of editors who can confidently make a prediction regarding its eventual unsuitability for acceptance (Fig. 1). Such a rejection requires the concurrence of at least two associate editors and the editor. All other submissions are sent out to two expert reviewers who

J. Clin. Invest.

(c) The American Society for Clinical Investigation, Inc.

$0021-9738 / 94 / 05 / 1871 / 04 \$ 2.00$

Volume 93, May 1994, 1871-1874 have agreed to evaluate the work. However, unlike the traditional process, these reviewers have the option of carrying out a rapid screening rejection, in place of a full review, on manuscripts that are felt to be in the bottom $50 \%$ (The Journal can eventually publish $<25 \%$ of all submissions). To aid this process, reviewers are provided with a checklist of potential reasons for an early rejection (Fig. 2), and space to provide brief remarks, if appropriate. If both reviewers return a screening rejection, the manuscript is returned to the authors with some comments regarding the reasons for the rejection. In the event of a split decision at screening the associate editor handling the manuscript can cast a deciding vote. In practice the associate editor usually errs on the side of supporting the authors for a full review, obtaining a third review if necessary (Fig. 1).

While this form of screening involves more work on the part of the editorial office and the reviewers, and may take significantly longer than an "editorial reject," it has several advantages, and is potentially fairer. The most important advantage is that the reviewer, who is an expert in the particular field, is more capable of rendering a well-informed judgment about the novelty, long term "importance," and broad "relevance" of the work at hand. Also, expert reviewers generally tend to be advocates of their own fields. Thus, a manuscript of high quality is less likely to be rejected just because an editor thinks that it is not "hot" or "popular" enough in its message. Conversely, this approach decreases the likelihood that poorly performed studies from currently competitive fields ( 8 ) will be given unwarranted attention over superior work from less popular fields. Further, since each manuscript will have been screened independently by three individuals (two expert reviewers and an associate editor), a single negative opinion at screening will not prevent a manuscript from eventually being accepted. There is one important additional benefit that is less obvious: because the screening review takes less time, this process recognizes the heavy burden of unrecompensed time commitment carried by the reviewer. The overall workload of the reviewers is reduced by this approach, allowing them to deal more efficiently with manuscripts that do merit complete review. Finally, the initial screening process will not delay the review of the eventually successful manuscript because it is already in the hands of the individuals who will provide the full review. In fact it might actually speed it up, because the requirement for immediate screening ensures that the reviewer takes a first look at the manuscript soon after receipt, rather than placing it under a pile of other pressing matters. These then were the theoretical reasons why the JCI embarked upon this novel approach to screening review. Since such a review process had never been tried before by any journal, the board felt it was important to evaluate the positive and negative consequences of the system at a relatively early date.

The screening review system: analysis of our experience to date. Overall, many more bouquets than brickbats have been tossed at the board since the inception of this system, giving the subjective impression that the experiment has been a success. The objective assessment of the outcome is by no means straightforward. This is because almost everyone involved has a biased view on the matter. As might be expected, most reviewers favor the system (because it saves them time and ef- 


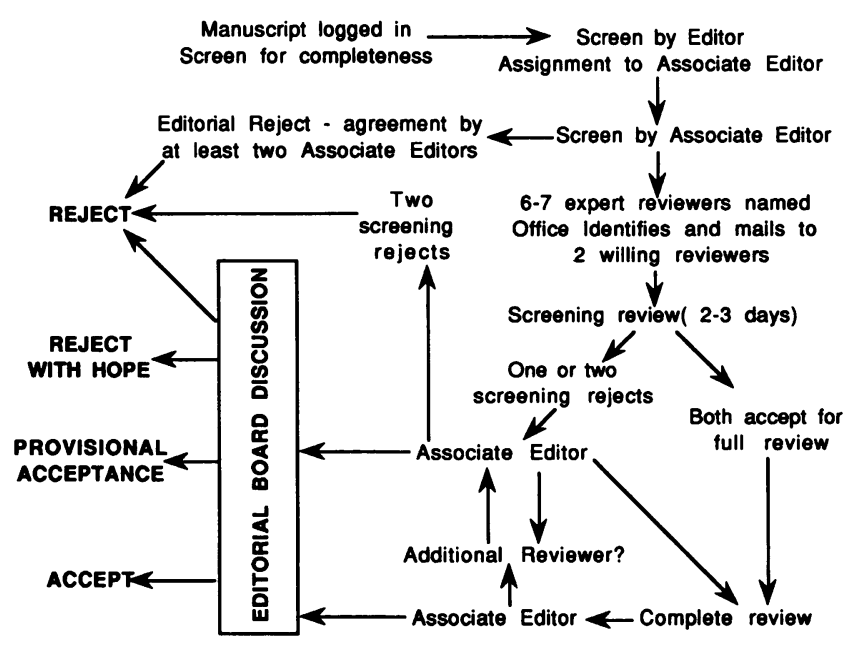

Figure 1. The current JCI manuscript review process. See text and references 3 and 4 for details.

fort), authors who are rejected intensely dislike it, and those whose papers are accepted are either indifferent to it, or like the fact that turnaround time has improved. The paradox, of course, is that there is a substantial overlap between our author and reviewer databases, i.e., many reviewers are authors and vice versa. Thus, it became evident that there was little point in polling authors and reviewers for their opinions on this matter. However, the Board did survey the opinions of members of the editorial committee and the consulting editors. Being spread throughout the world in numerous institutions, these individuals (whose names are listed on the masthead of The Journal) might also have received unsolicited feedback in one form or another. Of the Board members who responded to a formal poll, $88 \%$ concluded that the screening review process was a positive improvement and none indicated that it was worse than the traditional system. About half the respondents were also able to specifically comment about secondary feedback from others. Here too, the majority (68\%) indicated that their colleagues felt the system was a success, and only $2 \%$ reported strongly negative responses. Thus, from the subjective point of view, the screening review system appears to have been well received.

It is possible to obtain objective data regarding the practical mechanics of the new review process and its outcomes. To do this, we studied 931 consecutive regular manuscripts (excluding other categories such as Rapids, Perspectives, and Editorials) that underwent initial peer review from January through July 1993. As shown in Fig. 3, 11\% of these received an early editorial rejection, and $\sim 13 \%$ were declined on the basis of two screening rejects from reviewers. Of the $30 \%$ that received a split decision at screening (one reviewer returning a screening rejection and the other recommending a full review), nearly all were allowed to proceed to full review (i.e., giving the author the benefit of the doubt). However, as shown in Fig. 4, a screening rejection by one reviewer pointed to a very high probability $(\sim 70 \%)$ of rejection (at screening or after full review) by the second reviewer. Even in the instances where the second reviewer recommends acceptance, the priority ratings assigned are often not high. This engenders some confidence in the screening rejection process. The predictive value of a screening rejection is further demonstrated by the final outcome: $\sim 94 \%$ of all manuscripts that had one such rejection were eventually declined (see Fig. 3). Of course, it could be argued that the presence of a single screening rejection in the file might bias the editors against the manuscript, regardless of the outcome of the second review. However, against this is the fact that a small but significant number of such manuscripts were eventually pub-

\section{BASED UPON YOUR SCREENING OF THIS MANUSCRIPT, PLEASE CHECK THE APPROPRIATE BOX:}

\section{[ ] TOP 50\% Manuscript deserves a full review for possible acceptance.}

(Please fax this form to us in 2-3 days, then complete the enclosed blue and white forms for a full review and fax back within two weeks)

\begin{tabular}{l}
\hline [ ] BOTTOM 50\% Manuscript does not deserve a full detailed review for reasons \\
checked below. Any comments for Editors/Authors are listed below. \\
(Please destroy manuscript and return original figures) \\
[ ] Fatal flaws in overall logic or design. \\
[ ] Purely methodological report, or development of assay, with no application to a specific question. \\
[ ] Simple descriptive survey, with no specific question and no substantial discovery. \\
[ ] Single case report, without remarkable findings. \\
[ ] Findings are largely repetitive of previously published information.* \\
[ ] Findings represent minor incremental advance over previous information.* \\
[ ] Human homologue of a previously cloned gene/cDNA, with no unique or unusual features.* \\
with nopment remarkably of a new drug closely related to a previously described one, \\
[ ] Genetic abnormality/variation in the DNA sequence of a gene with many previously well \\
characterized mutations, and no new biological insights arising from the study.* \\
[ ] Other reasons, indicated below. \\
Comments for ause specify the precedent
\end{tabular}

Figure 2. The screening review form used by reviewers for initial decisions. 


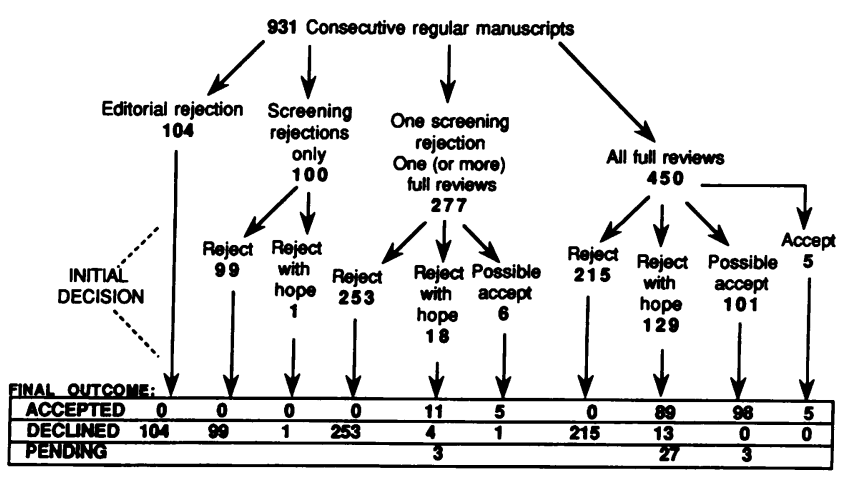

Figure 3. Initial decisions and final outcomes on 931 consecutive regular articles. The data are presented relative to the initial recommendations for editorial rejection, screening rejection, or full review.

lished. Thus, a malicious reviewer intent on deliberately sabotaging a competitors article cannot be sure that a screening reject will "blackball" the article. Those who argue that the screening process still provides an opportunity for such attempted sabotage must realize that the situation would be no different in the regular review process. Thus, the rare reviewer who is intent on mischief could accomplish this just as effectively (with more loss of time for the author) by an in-depth protracted negative review.

What effect has the new review process had on the time from submission to first decision? As shown in Fig. 5, editorial rejections were rendered with an average of 8.9 days. This appears to be a reasonable time frame, since such a rejection requires the agreement of at least two associate editors, or a discussion at the weekly editorial board meeting. The small "tail" in this curve is due to the fact that some editorial rejections require the additional opinions of a third associate or consulting editor. With regard to manuscripts that received a screening rejection (see Fig. 5), the mean time to decision was 31 days, with $\sim 80 \%$ occurring within 40 days. The small but very significant tail on this profile is mostly explained by manuscripts for which great difficulties were encountered in obtaining willing reviewers (discussed below). It is understandable

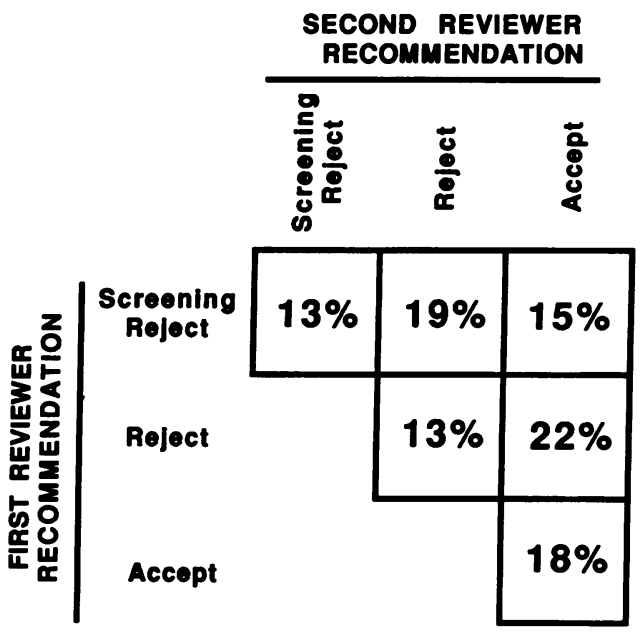

Figure 4. Correlation between first and second reviewer recommendations. The percentages in all of the boxes add up to $100 \%$, and include all manuscripts from the current set of 931 that received two separate reviews.

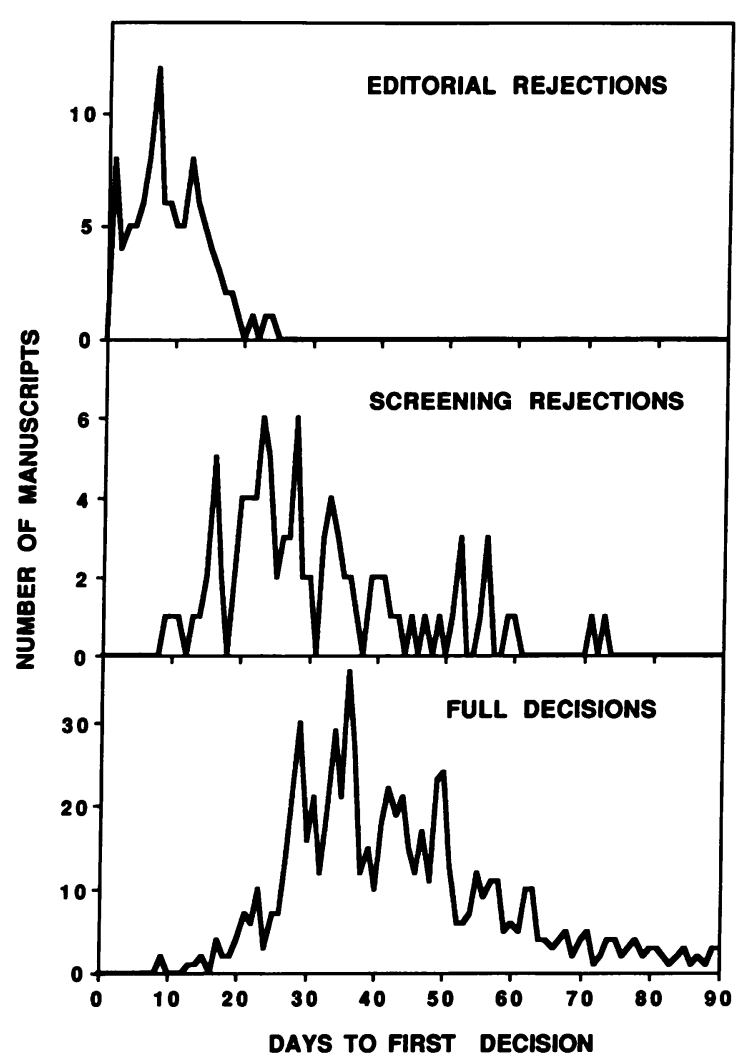

Figure 5. Days to first decision on 931 consecutive regular articles. Frequency distribution of time from receipt of manuscripts to mailing of first decision is indicated for the three categories of editorial rejects, screening rejects, and full decisions. A few manuscripts that took $>90$ days are not shown.

that authors might be unhappy about receiving a "screening rejection" after more than 40 days have elapsed. However, asking the late-identified reviewers on such manuscripts to not avail themselves of the screening option might only delay the review further, with the same negative outcome being likely. This relatively rare occurrence remains an unresolved problem in the present system.

Effects of the new system on the overall review process. With regard to manuscripts that proceeded to a full review (from one or both of the reviewers), the time to first decision was spread over a wide range ( see Fig. 5) with a mean of 45 days, and with $>75 \%$ of decisions being rendered by 60 days. While this represents a substantial improvement over prior years (see below) the persistence of a small but significant tail beyond 65 days is clearly evident. The main reasons for these late decisions are reviewer-related delays. The most common instance occurs when most or all of the reviewers who are contacted refuse the opportunity to review the manuscript (this might mean either that no one is interested in the work, that the work is in a narrow specialty field with few experts, or that the field of work is so topical and popular, that all available reviewers are busy!). In other cases, a reviewer who had originally agreed to review the manuscript may take inordinately long to do so, or even change his/her mind about doing the review at all. While the editorial office makes every effort to avoid these situations, authors must recognize that the reviewers do a completely voluntary and unrecompensed service. Thus, while the editorial office requests that reviewers be prompt, and reminds them when they are tardy, it is the reviewer who has the ultimate 


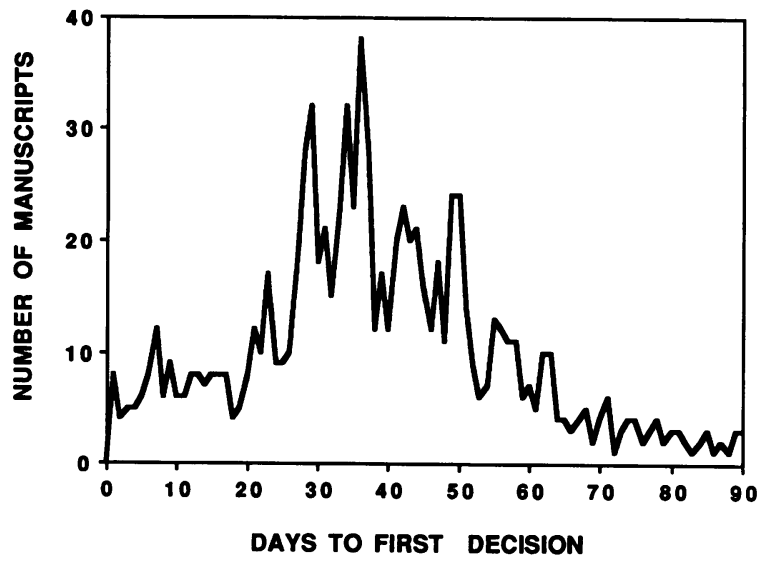

Figure 6. Days to first decision-the overall profile. Frequency distribution of time from receipt of manuscripts to mailing of the first decision for all 931 regular manuscripts studied in this report (includes all those presented in Fig. 5). A few manuscripts that took $>90$ days are not shown.

responsibility of responding. In such cases, editors do consider the possibility that the reviewer is deliberately delaying the process for malicious reasons, and may elect to obtain another review or to review the manuscript themselves-unfortunately this may result in a further delay.

The institution of this new system and the associated streamlining (use of overnight mail, fax and full computerization) has yielded significant improvements in overall review time. Fig. 6 shows the overall profile of decision times for the 931 regular manuscripts studied here. The overall average handling time for this group was 43 days, with $>60 \%$ of decisions being rendered in 45 days. It is of note that this average time for handling is not very far from that predicted for the "ideal" manuscript, for which every possible step proceeds perfectly ( see Table I). In comparison, average handling time for a comparable group of regular manuscripts in 1991 was 51 days, with only $36 \%$ of the decisions being rendered in 45 days (detailed data not shown). All this may be small comfort to the few authors whose manuscripts happen to fall into the persistent tail of the "very late" ( $>65$ days) category. However, they must realize that since manuscript handling (like any other complex process) is affected by many variables, it is unlikely that this tail will ever be completely eliminated.

A few authors have written to complain that the screening review system has diminished or even eliminated something they once looked for from The Journal - detailed constructive reviews which, even if negative, provided them advice for their future work. While this is indeed one potential side benefit of the peer review system, the primary purpose of The Journal is not to provide authors with detailed feedback regarding the flaws in their work and advice for future studies. Rather, it is to attempt to publish the best possible collection of suitable original articles following fair and expedient peer review.

No system of peer review can claim to be completely fair, and this one is no exception. Thus, rebuttals of decisions by authors are always taken very seriously, and handled in a uniform manner outlined elsewhere $(3,4)$, being discussed in detail by the editorial board, and sometimes generating additional reviews or opinions from consulting editors. Among the 931 consecutive manuscripts reported on here, 43 negative decisions generated rebuttals. After careful examination, 39 of
Table I. "Ideal" Timelines for Handling of a Manuscript

\begin{tabular}{|c|c|c|}
\hline \multirow[b]{2}{*}{ Step } & \multicolumn{2}{|c|}{ Number of days } \\
\hline & Full decision & Screening reject \\
\hline Receipt and check in & 1 & 1 \\
\hline Assignment to associate editor & 1 & 1 \\
\hline Assignment of reviewers* & 1 & 1 \\
\hline Fax to reviewers & 1 & 1 \\
\hline Acceptance by reviewers ${ }^{\ddagger}$ & 2 & 2 \\
\hline Mail out to reviewers $\$$ & 1 & 1 \\
\hline Review faxed by reviewers" & 14 & 2 \\
\hline To associate editor* & 1 & 1 \\
\hline Presentation at board meeting' & 3 & - \\
\hline Decision letter generated & 1 & 1 \\
\hline Decision reviewed and signed & 1 & 1 \\
\hline Decision logged and mailed & 1 & 1 \\
\hline Total time & 28 days & 13 days \\
\hline
\end{tabular}

\footnotetext{
* Assumes that the associate editor is not away, and that the choice of reviewers is straightforward.

${ }^{\ddagger}$ Assumes that the first two potential reviewers contacted promptly accept the assignment.

$\$$ By overnight mail. Assumes North American reviewer.

"Assumes average time to next weekly editorial board meeting.

"Assumes that the reviewers respond within the recommended time.
}

these rebuttals resulted in no change of the original decision (strengthening the feeling that the process is generally sound). However, four rebuttals $(\sim 10 \%)$ were successful in generating requests for resubmission, and of these, two eventually resulted in acceptance.

Conclusions. Based on both the objective facts and the subjective information described above, the editorial board has concluded that the screening review system is worthy of continuation in its present form. If imitation is indeed the sincerest form of flattery, it is noteworthy that several other journal offices have inquired about the mechanics of the process, and that some have actually instituted similar review systems. However, all editorial boards that choose to try this new process are well advised to monitor it carefully, and to verify that it does indeed result in the fairest possible review for each individual manuscript submitted to them. The JCI board intends to do so.

Ajit P. Varki

Editor

for the Editorial Board

\section{References}

1. Scharschmidt, B. F. 1990. Something old, something new, something blue. J. Clin. Invest. 85:1.

2. Varki, A. 1992. The times they are a'changin. Changing with the times. $J$. Clin. Invest. 89:721-722.

3. Varki, A. 1993. Editorial policies and practices of The Journal of Clinical Investigation. J. Clin. Invest. 92:v-vii.

4. Varki, A. 1994. Editorial policies and practices of The Journal of Clinical Investigation. J. Clin. Invest. 93:v-vii.

5. Wilson, J. D. 1978. Peer review and publication. J. Clin. Invest. 61:16971701.

6. Stossel, T. P. 1985. Refinement in biomedical communication: a case study. In Science, Technology, and Human values. John Wiley \& Sons, New York. 10:39-43.

7. Koshland, D. 1985. An Editor's quest II. Science (Wash. DC). 227:249.

8. Maddox, J. 1993. Competition and the death of science. Nature (Lond.). 363:667. 